\title{
SEMIGROUPS THROUGH SEMILATTICES
}

\author{
BY \\ J. H. CARRUTH AND JIMMIE D. LAWSON
}

\begin{abstract}
Presented in this paper is a method of constructing a compact semigroup $S$ from a compact semilattice $X$ and a compact semigroup $T$ having idempotents contained in $X$. The notions of semigroups (straight) through chains and (straight) through semilattices are introduced. It is shown that the notion of a semigroup through a chain is equivalent to that of a generalized hormos. Universal objects are obtained in several categories including the category of clans straight through a chain and the category of clans straight through a semilattice relative to a chain. An example is given of a nonabelian clan $S$ with abelian set of idempotents $E$ such that $S$ is minimal (as a clan) about $E$.
\end{abstract}

1. Introduction. In their book Elements of compact semigroups K. H. Hofmann and P. S. Mostert present a technique for constructing a compact topological semigroup called a generalized hormos beginning with a totally ordered compact chain. Hofmann and Mostert raise the question of whether the compact chain can be replaced by a compact topological semilattice [2, p. 160]. The purpose of this paper is to present one method for constructing semigroups from such semilattices.

In $\S 3$ we give a definition of a semigroup through a chain and prove that the class of such semigroups coincides with the class of generalized hormi.

In $\$ 4$ we generalize the concept of a semigroup through a chain to the concept of a semigroup through a semilattice. Such semigroups consist of a generalized hormos through a chain in the semilattice united with homomorphic images of the generalized hormos through translates of the chain. Although we do not give a general construction for all semigroups through a semilattice, we do construct a universal one for a fixed semilattice and generalized hormos.

In $\S 5$ we consider a slightly different class of semigroups and again construct a universal semigroup in this class.

Commutative semigroups which are irreducible about their idempotents are examples of semigroups through semilattices. We briefly discuss this class of examples in $\$ 6$.

2. Preliminaries. Throughout this paper $\leqq$ will denote the $\mathscr{H}$ quasi-order on a semigroup $S$, i.e. $a \leqq b$ if and only if $S^{1} a \subset S^{1} b$ and $a S^{1} \subset b S^{1}$. The set of elements which are $\mathscr{H}_{-}\left(\mathscr{D}_{-}\right)$equivalent to a fixed $x \in S$ will be denoted by $H(x)(D(x))$.

Received by the editors March 31, 1970.

AMS 1969 subject classifications. Primary 2205; Secondary 2092, 2093.

Key words and phrases. Compact semigroup, compact semilattice, clan, irreducible semigroup, generalized hormos, universal object. 
Definitions of the $\mathscr{D}$ and $\mathscr{H}$ relations are given in [2, p. 29]. If $y \in S$, we set

$$
L(y)=\{z \in S: z \leqq y\}, \quad U(y)=\{z \in S: y \leqq z\} .
$$

We note that if $S$ is a semilattice (a commutative, idempotent semigroup), then the $\mathscr{H}$ quasi-order coincides with the natural partial order on $S$ defined by $e \leqq f$ if and only if $e f=e$.

If $S$ is a semigroup, we denote the set of idempotents of $S$ by $E(S)$. An iseomorphism is a mapping from a topological semigroup $S$ into a topological semigroup $S^{\prime}$ which is both an isomorphism and a homeomorphism. A clan is a compact, connected topological semigroup with identity.

3. A characterization of generalized hormi. Throughout this section only $X$ will denote a compact, totally ordered topological semilattice with maximal element 1 and minimal element $z$.

Definition 3.1. The pair $(S, \phi)$ is a semigroup through $X$ if $S$ is a compact topological semigroup with identity and $\phi$ is an iseomorphism from $X$ into $S$ satisfying

(a) $\phi(1)$ is the identity of $S$ and $\phi(z)$ is in the minimal ideal of $S$,

(b) $S=L(f) \cup U(f)$ for each $f \in \phi(X)$.

If, in addition, $\phi(X)=E(S)$, then $(S, \phi)$ is a semigroup straight through $X$. If $S$ is connected, then $(S, \phi)$ is a clan through $X$.

Definition 3.2. If $\left(S_{1}, \phi_{1}\right)$ and $\left(S_{2}, \phi_{2}\right)$ are semigroups through $X$, then a homomorphism from $\left(S_{1}, \phi_{1}\right)$ into $\left(S_{2}, \phi_{2}\right)$ is a homomorphism $\alpha$ from $S_{1}$ into $S_{2}$ satisfying $\alpha \phi_{1}=\phi_{2}$. If $\alpha$ is an iseomorphism onto $S_{2}$, then $\left(S_{1}, \phi_{1}\right)$ and $\left(S_{2}, \phi_{2}\right)$ are iseomorphic.

In some cases where no ambiguity arises, no explicit mention is made of $\phi$ and $S$ is referred to simply as a semigroup through $X$. In this case we denote $\phi(x)$ by $e_{x}$.

We now show that the concepts of a generalized hormos and a semigroup through a chain are the same. We begin with the following lemma:

LEMMA 3.1. Let $S$ be a semigroup through $X$. Then

(1) for each $x \in X, D\left(e_{x}\right)=H\left(e_{x}\right)$ and $e_{x}$ is in the centralizer of $S$;

(2) for each $x, y \in X$ such that $x \leqq y, L\left(e_{y}\right) \cap U\left(e_{x}\right)$ is a compact subsemigroup of $S$ with identity $e_{y}$, group of units $H\left(e_{y}\right)$, and minimal ideal $H\left(e_{x}\right)$.

Proof. That $D\left(e_{x}\right)=H\left(e_{x}\right)$ follows immediately from $U\left(e_{x}\right) \cup L\left(e_{x}\right)=S$. If $y \in U\left(e_{x}\right)$, then $y e_{x}, e_{x} y \in D\left(e_{x}\right)=H\left(e_{x}\right)$. Hence $y e_{x}=e_{x} y e_{x}=e_{x} y$. If $y \in L\left(e_{x}\right)$, then $e_{x} y=y=y e_{x}$. Hence $e_{x}$ is in the centralizer of $S$.

If $r, s \in U\left(e_{x}\right)$, then $e_{x} r, e_{x} s \in H\left(e_{x}\right)$. Thus $e_{x} r e_{x} s=e_{x} r s \in H\left(e_{x}\right)$ and so $r s \in U\left(e_{x}\right)$. Since $L\left(e_{y}\right)=e_{y} S e_{y}, L\left(e_{y}\right)$ is also a semigroup with identity $e_{y}$. Hence $L\left(e_{y}\right) \cap U\left(e_{x}\right)$ is a semigroup with identity $e_{y}$ and group of units $H\left(e_{y}\right)$. Since $w e_{x}, e_{x} w \in H\left(e_{x}\right)$ for every $w \in U\left(e_{x}\right)$, we conclude that $H\left(e_{x}\right)$ is the minimal ideal of $L\left(e_{y}\right) \cap U\left(e_{x}\right)$.

In the following all references will be to Hofmann and Mostert [2]. The omitted definitions and terminology may be found therein. 
THEOREM 3.1. Let $X$ be a compact, totally ordered topological semilattice. Then there exists an iseomorphism $\phi$ such that $(S, \phi)$ is a semigroup through $X$ if and only if $S$ is a generalized hormos with totally ordered space $X$.

Proof. Assume that $(S, \phi)$ is a semigroup through $X$. We set $X^{\prime}=\{x \in X:[y, x]$ $=\{y, x\}$ for some $y<x\}$. The unique element $y$ with this property is denoted by $x^{\prime}$. For each $x \in X^{\prime}$, we set $S_{x}^{\prime}=L(\phi(x)) \cap U\left(\phi\left(x^{\prime}\right)\right)$. For each $x \notin X^{\prime}$, we set $S_{x}^{\prime}=H(\phi(x))$. For each $x, y \in X$ with $x \leqq y$ we define $m_{x y}^{\prime}(s)=\phi(x) s$. Finally, we set $S_{x}=x \times S_{x}^{\prime}$ and define $m_{x y}$ as a suitable modification of $m_{x y}^{\prime}$. It follows from (1) of Lemma 3.1 that $m_{x y}$ is a homomorphism and from (2) that $S_{x}$ is a compact semigroup with identity $\phi(x)$.

By techniques which closely parallel those of Hofmann and Mostert (see [2, 5.7, pp. 143-144]), one can show that $\left(X, S_{x}, m_{x y}\right)$ is a chainable collection and that the function from GHorm $\left(X, S_{x}, m_{x y}\right)$ onto $S$ defined by $f((x, s))=s$ is an iseomorphism.

Conversely, suppose $S=\operatorname{GHorm}\left(X, S_{x}, m_{x y}\right)$. Define $\phi$ from $X$ into $S$ by $\phi(x)=u_{x}$, where $u_{x}$ is the identity of $S_{x}$. Then $\phi$ is an iseomorphism, $\phi(1)=1$, and $\phi(z)$ is in the minimal ideal of $S$. If $t \in S_{y}$ for $x<y$, then $t u_{x}$ and $u_{x} t$ are elements of $H_{x}=H\left(u_{x}\right)$, and hence $u_{x} \leqq t$. If $t \in S_{z}$ for some $z \leqq x$, then $u_{x} t=u_{z} u_{x} t=u_{z} t=t$. Similarly $t u_{x}=t$ and hence $t \leqq u_{x}$. Thus if $u_{x} \in \phi(X)$, then $S=L\left(u_{x}\right) \cup U\left(u_{x}\right)$. Hence $(S, \phi)$ is a semigroup through $X$.

The iseomorphism $\phi$ defined in the second part of this proof is called the natural mapping of $X$ into GHorm $\left(X, S_{x}, m_{x y}\right)$.

Theorem 3.1 sometimes provides a convenient formulation for generalized hormi.

COROLLARY 3.1.1. The homomorphic image of a generalized hormos is a generalized hormos.

Proof. Let $S$ be a generalized hormos and let $g$ be a continuous homomorphism from $S$ onto $T$. By Theorem 3.1 there exists an iseomorphism $\phi$ from $X$ into $S$ such that ${ }^{-}(S, \phi)$ is a semigroup through $X$. We set $Y=\{g \phi(x): x \in X\}$. If $i$ denotes the injection of $Y$ into $T$, then it is easily checked that $(T, i)$ is a semigroup through $Y$. Hence by Theorem $3.1 T$ is a generalized hormos with totally ordered space $Y$.

4. Construction of universal semigroups through a semilattice. Throughout the remainder of this paper $X$ will denote a compact topological semilattice with identity 1 and zero $z$ and $C$ will be a closed chain in $X$ containing 1 and $z$.

Definition 4.1. The triple $\left(S, S^{\prime}, \psi\right)$ is a semigroup through $X$ relative to $C$ if $S$ is a compact topological semigroup with identity, $S^{\prime}$ is a closed subsemigroup containing the identity, and $\psi$ is an iseomorphism from $X$ into $S$ satisfying

(a) $\left(S^{\prime}, \psi \mid C\right)$ is a semigroup through $C$,

(b) $\psi(X)$ is contained in the centralizer of $S^{\prime}$,

(c) $S=\psi(X) \cdot S^{\prime}$. 
If in addition $\psi(X)=E(S)$ and $\psi(C)=E\left(S^{\prime}\right)$, then $\left(S, S^{\prime}, \psi\right)$ is a semigroup straight through $X$ relative to $C$. The semigroup $\left(S, S^{\prime}, \psi\right)$ through $X$ relative to $C$ is a clan through $X$ relative to $C$ if $S$ and $S^{\prime}$ are connected.

Definition 4.2. If $\left(S_{1}, S_{1}^{\prime}, \psi_{1}\right)$ and $\left(S_{2}, S_{2}^{\prime}, \psi_{2}\right)$ are semigroups through $X$ relative to $C$, then a homomorphism from $\left(S_{1}, S_{2}^{\prime}, \psi_{1}\right)$ into $\left(S_{2}, S_{1}^{\prime}, \psi_{2}\right)$ is a homomorphism $\alpha$ from $S_{1}$ into $S_{2}$ such that $\alpha \mid S_{1}^{\prime}$ is a homomorphism from $\left(S_{1}^{\prime}, \psi_{1} \mid C\right)$ into $\left(S_{2}^{\prime}, \psi_{2} \mid C\right)$ and the following diagram is commutative:

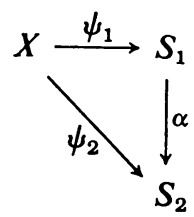

Definition 4.3. Let $(T, \phi)$ be a fixed semigroup through $C$. We will denote by $\mathscr{C}(X, C, T, \phi)$ the category whose objects are semigroups $\left(S, S^{\prime}, \psi\right)$ through $X$ relative to $C$ such that $\left(S^{\prime}, \psi \mid C\right)$ and $(T, \phi)$ are iseomorphic. The morphisms from $\left(S_{1}, S_{1}^{\prime}, \psi_{1}\right)$ to $\left(S_{2}, S_{2}^{\prime}, \psi_{2}\right)$ are homomorphisms $\alpha$ from $\left(S_{1}, S_{1}^{\prime}, \psi_{1}\right)$ into $\left(S_{2}, S_{2}^{\prime}, \psi_{2}\right)$ such that $\alpha \mid S_{1}^{\prime}$ is an iseomorphism from $\left(S_{1}^{\prime}, \psi_{1} \mid C\right)$ onto $\left(S_{2}^{\prime}, \psi_{2} \mid C\right)$.

Definition 4.4. A universal object in a category $\mathscr{C}$ is an object $X$ of $\mathscr{C}$ such that for every object $Y$ of $\mathscr{C}, \operatorname{hom}(X, Y)$ is nonvoid.

RemarKs. In view of Definition 4.1 (c), each morphism in the category $\mathscr{C}(X, C, T, \phi)$ is a surmorphism and hence an epimorphism. Therefore, a universal object in $\mathscr{C}(X, C, T, \phi)$ is a semigroup $\left(S, S^{\prime}, \psi\right)$ through $X$ relative to $C$ with $\left(S^{\prime}, \psi \mid C\right)$ iseomorphic to $(T, \phi)$ such that if $\left(S_{1}, S_{1}^{\prime}, \psi_{1}\right)$ is any other such, then there exists a homomorphism $\alpha$ from $\left(S, S^{\prime}, \psi\right)$ onto $\left(S_{1}, S_{1}^{\prime}, \psi_{1}\right)$ such that $\alpha \mid S_{1}^{\prime}$ is an iseomorphism from $\left(S^{\prime}, \psi \mid C\right)$ onto $\left(S_{1}^{\prime}, \psi_{1} \mid C\right)$. The proof of the next theorem can be considered as a method of constructing a semigroup from a semilattice $X$ and a generalized hormos $T$ by joining each $x \in X$ to $z$ by a homomorphic image of $T$ (which is again a generalized hormos by Corollary 3.1.1). In fact, one needs only to start with a semilattice and a chainable collection as the two corollaries indicate.

THEOREM 4.1. If $(T, \phi)$ is a fixed semigroup through $C$ then there exists a universal object in the category $\mathscr{C}(X, C, T, \phi)$.

Corollary 4.1.1. Let $\mathscr{C}=\left\{C, S_{x}, m_{x y}\right\}$ be a chainable collection and let $\phi$ be the natural map from $C$ into $\operatorname{GHorm}\left(C, S_{x}, m_{x y}\right)$. Then there exists a semigroup $\left(S, S^{\prime}, \psi\right)$ through $X$ relative to $C$ such that $\left(S^{\prime}, \psi \mid C\right)$ is iseomorphic to (GHorm $\left.\left(C, S_{x}, m_{x y}\right), \phi\right)$.

Corollary 4.1.2. Let $\mathscr{C}=\left\{C, S_{x}, m_{x y}\right\}$ be a chainable collection such that $S_{x}$ is cylindrical for each $x \in C$ and let $\phi$ be the natural map from $C$ into $\operatorname{Horm}\left(C, S_{x}, m_{x y}\right)$. 
Then there exists a semigroup $\left(S, S^{\prime}, \psi\right)$ straight through $X$ relative to $C$ such that $\left(S^{\prime}, \psi \mid C\right)$ is iseomorphic to (Horm $\left.\left(C, S_{x}, m_{x y}\right), \phi\right)$.

Proof of Theorem 4.1. The method of proof is to define a congruence $\mathscr{C}$ on the semigroup $X \times T$ in such a way that the quotient semigroup $(X \times T) / \mathscr{C}$ has the desired properties. For convenience we consider $C$ as a subset of $T$ by identifying $C$ with its image under $\phi$ in $T$. Let $\mathscr{P}$ and $\mathscr{Q}$ be the binary relations on $X \times T$ defined by

$$
\begin{gathered}
\mathscr{P}=\left\{((f, r),(g, s)): \text { for some } a_{1}, a_{2}, a_{3} \in C, a_{1} \leqq r \leqq a_{2},\right. \\
\left.a_{1} \leqq s \leqq a_{3}, a_{1} f=a_{2} f=a_{1} g=a_{3} g, \text { and } a_{1} r=a_{1} s\right\}, \\
\mathscr{Q}=\{((f, r),(g, s)): r=s \text { and for some } a \in C, r \leqq a \text { and } a f=a g\} .
\end{gathered}
$$

Let $\mathscr{C}=\mathscr{P} \cup \mathscr{Q}$. We proceed to show that $\mathscr{C}$ is a closed congruence on $X \times T$.

Simple subnet arguments using the facts that $C$ and the graph of $\leqq$ are closed yield that both $\mathscr{P}$ and $\mathscr{Q}$ are closed; hence $\mathscr{C}$ is closed. Since $C$ contains $1, \mathscr{Q}$ is reflexive and hence $\mathscr{C}$ is. That $\mathscr{C}$ is symmetric follows from the obvious fact that both $\mathscr{P}$ and $\mathscr{Q}$ are symmetric. Next we show that $\mathscr{C}$ is transitive.

Fix $((f, r),(g, s))$ and $((g, s),(h, t))$ in $\mathscr{C}$. That $((f, r),(h, t))$ is in $\mathscr{C}$ will be verified by examining the following four cases:

Case 1. $((f, r),(g, s)) \in \mathscr{P}$ and $((g, s),(h, t)) \in \mathscr{P}$. In this case there exist $a_{1}, a_{2}, a_{3}, b_{1}, b_{2}, b_{3} \in C$ such that $a_{1} \leqq r \leqq a_{2}, a_{1} \leqq s \leqq a_{3}, b_{1} \leqq s \leqq b_{2}, b_{1} \leqq t \leqq b_{3}$, $a_{1} f=a_{2} f=a_{1} g=a_{3} g, b_{1} g=b_{2} g=b_{1} h=b_{3} h, a_{1} r=a_{1} s$, and $b_{1} s=b_{1} t$. Let $c_{1}=a_{1} b_{1}$, $c_{2}=a_{2}$, and $c_{3}=b_{3}$. Since $a_{1} b_{1}=\min \left\{a_{1}, b_{1}\right\}$ we have $c_{1} \leqq r \leqq c_{2}$ and $c_{1} \leqq t \leqq c_{3}$. Now,

$$
\begin{aligned}
& c_{1} f=a_{1} b_{1} f=b_{1} a_{1} f=b_{1} a_{1} g=a_{1} b_{1} g \\
& c_{2} f=a_{2} f=a_{1} g=a_{1} b_{2} g \text { since } a_{1} \leqq s \leqq b_{2} \\
& =a_{1} b_{1} g \\
& c_{1} h=a_{1} b_{1} h=a_{1} b_{1} g \\
& c_{3} h=b_{3} h=b_{1} g=b_{1} a_{3} g \text { since } b_{1} \leqq s \leqq a_{3} \\
& =b_{1} a_{1} g \\
& =a_{1} b_{1} g \text {. }
\end{aligned}
$$

Hence, $c_{1} f=c_{2} f=c_{1} h=c_{3} h$. Finally, $a_{1} r=a_{1} s$ implies $a_{1} b_{1} r=a_{1} b_{1} s$ and $b_{1} s=b_{1} t$ implies $a_{1} b_{1} s=a_{1} b_{1} t$. Therefore, $c_{1} r=a_{1} b_{1} r=a_{1} b_{1} t=c_{1} t$ and it follows that $((f, r),(h, t)) \in \mathscr{P}$.

Case 2. $((f, r),(g, s)) \in \mathscr{Q}$ and $((g, s),(h, t)) \in \mathscr{Q}$. Here $r=s=t$ and there exist $a, b \in C$ such that $r \leqq a, r \leqq b, a f=a g$, and $b g=b h$. Let $c=a b$. Then $r \leqq c$ and $c f=c h$ so that $((f, r),(h, t)) \in \mathscr{Q}$.

Case 3. $((f, r),(g, s)) \in \mathscr{P}$ and $((g, s),(h, t)) \in \mathcal{Q}$. There exist $a_{1}, a_{2}, a_{3}, a \in C$ such that $a_{1} \leqq r \leqq a_{2}, a_{1} \leqq s \leqq a_{3}, a_{1} f=a_{2} f=a_{1} g=a_{3} g, a_{1} r=a_{1} s, \quad s=t \leqq a$, and 
$a g=a h$. Let $b_{1}=a_{1}, b_{2}=a_{2}$, and $b_{3}=a_{3} a$. Then $b_{1} \leqq r \leqq b_{2}$ and $b_{1} \leqq s \leqq b_{3}$. Now,

$$
\begin{aligned}
& \begin{aligned}
b_{1} f=b_{2} f & =a_{1} g \\
b_{1} h=a_{1} h & =a_{1} a_{3} a h \quad \text { since } a_{1} \leqq s \leqq a_{3} a \\
& =a_{1} a_{3} a g \\
& =a_{1} g
\end{aligned} \\
& b_{3} h=a_{3} a h=a_{3} a g=a a_{1} g=a_{1} g .
\end{aligned}
$$

Hence, $b_{1} f=b_{2} f=b_{1} h=b_{3} h$. Finally $b_{1} r=a_{1} r=a_{1} t=b_{1} t$ so that $((f, r),(h, t)) \in \mathscr{P}$.

Case 4. $((f, r),(g, s)) \in \mathscr{Q}$ and $((g, s),(h, t)) \in \mathscr{P}$. It was shown in Case 3 that $\mathscr{P} \circ \mathscr{Q} \subset \mathscr{P}$. Therefore, $\mathscr{Q} \circ \mathscr{P}=\mathscr{Q}^{-1} \circ \mathscr{P}^{-1}=(\mathscr{P} \circ \mathscr{Q})^{-1} \subset \mathscr{P}-1=\mathscr{P}$ and it follows that $((f, r),(h, t)) \in \mathscr{P}$.

In order to complete the proof that $\mathscr{C}$ is a congruence, we show that $\Delta \mathscr{C} \cup \mathscr{C} \Delta \subset \mathscr{C}$. Fix $((f, r),(g, s)) \in \mathscr{P}$ and $(h, t) \in X \times T$. Then there exist $a_{1}, a_{2}, a_{3} \in C$ such that $a_{1} \leqq r \leqq a_{2}, a_{1} \leqq s \leqq a_{3}, a_{1} f=a_{2} f=a_{1} g=a_{3} g$, and $a_{1} r=a_{1} s$. Hence we have that $a_{1} r t=a_{1} s t$.

If $t \leqq a_{1}$, then $a_{1} t=t$. Since $a_{1}$ is in the centralizer of $T$ (Lemma 3.1), we have that $r t=r a_{1} t=a_{1} r t=a_{1} s t=s a_{1} t=s t$. Letting $a=a_{1}$, we conclude that $r t=s t, r t \leqq a$ since $t \leqq a_{1}$, and $a f h=a_{1} f h=a_{1} g h=a g h$. Thus $((f h, r t),(g h, s t)) \in \mathscr{Q}$.

If $a_{1} \leqq t$, then $a_{1} \leqq r t \leqq a_{2}$ and $a_{1} \leqq s t \leqq a_{3}$ (Lemma 3.1). Clearly $a_{1} f h=a_{2} f h$ $=a_{1} g h=a_{3} g h$ and $a_{1} r t=a_{1} s t$. Therefore $((f h, r t),(g h, s t)) \in \mathscr{P}$. Thus we conclude that $\mathscr{P} \Delta \subset \mathscr{P} \cup \mathscr{Q}=\mathscr{C}$. Using the fact that $C$ is in the centralizer of $T$, we can show similarly that $\Delta \mathscr{P} \subset \mathscr{C}$.

Now fix $((f, r),(g, r)) \in \mathscr{Q}$ and $(h, t) \in X \times T$. There exists $a \in C$ such that $r \leqq a$ and $a f=a g$. Hence $a f h=a g h$; we have that $r t \leqq a$ since $r \leqq a$. Thus $((f h, r t),(g h, r t))$ $\in \mathscr{Q} \subset \mathscr{C}$. That $\Delta \mathscr{Q} \subset \mathscr{Q} \subset \mathscr{C}$ follows by a similar argument using the fact that $X$ is commutative.

Now, let $S=(X \times T) / \mathscr{C}$ and let $\alpha$ be the natural homomorphism taking $X \times T$ onto $S$. Let $S^{\prime}=\alpha(\{1\} \times T)$. Define $\beta: X \rightarrow X \times T$ by $\beta(f)=(f, 1)$ and let $\psi=\alpha \circ \beta$. We proceed to show that $\left(S, S^{\prime}, \psi\right)$ is a semigroup through $X$ relative to $C$ such that $\left(S^{\prime}, \psi \mid C\right)$ is iseomorphic to $(T, \phi)$.

Obviously, $S$ is a compact topological semigroup with identity [2, page 19, Theorem 2.11] and $S^{\prime}$ is a compact subsemigroup of $S$ containing the identity of $S$. Since $\psi$ is the composition of two continuous homomorphisms, it is a continuous homomorphism. To see that $\psi$ is actually an iseomorphism, suppose $\psi(f)=\psi(g)$. Then, $((f, 1),(g, 1)) \in \mathscr{C}$. In the case that $((f, 1),(g, 1)) \in \mathscr{P}$ there exist $a_{1}, a_{2}, a_{3} \in C$ such that $a_{1} \leqq 1 \leqq a_{2}, a_{1} \leqq 1 \leqq a_{3}$, and $a_{1} f=a_{2} f=a_{1} g=a_{3} g$. But $1 \leqq a_{2}$ and $1 \leqq a_{3}$ imply $a_{2}=a_{3}=1$, so that $f=g$. If $((f, 1),(g, 1)) \in \mathscr{Q}$ then there exists $a \in C$ such that $a \geqq 1$ and $a f=a g$. Again $a \geqq 1$ implies $a=1$ so that $f=g$. Hence, $\psi$ is an iseomorphism from $X$ into $S$.

Next we show that $\left(S^{\prime}, \psi \mid C\right)$ is a semigroup through $C$ and that $\left(S^{\prime}, \psi \mid C\right)$ is iseomorphic to $(T, \phi)$. Define $\delta: T \rightarrow S^{\prime}$ by $\delta(r)=\alpha(1, r)$. Clearly, $\delta$ is a continuous 
homomorphism from $T$ onto $S^{\prime}$. Recalling that $C$ is identified with $\phi(C)$ in $T$, $(\delta \circ \phi)(e)=\delta(\phi(e))=\alpha(1, \phi(e))=\alpha(1, e)$, whereas $(\psi \mid C)(e)=(\alpha \circ \beta)(e)=\alpha(e, 1)$ for each $e \in C$. For a fixed $e \in C$ let $a_{1}=e, a_{2}=e$, and $a_{3}=1$. Then $a_{1} \leqq e \leqq a_{2}, a_{1} \leqq 1 \leqq a_{3}$, $a_{1} 1=a_{2} 1=a_{1} e=a_{3} e$, and $a_{1} e=a_{1} 1$, so that $((1, e),(e, 1)) \in \mathscr{P}$. Therefore, $\alpha(1, e)$ $=\alpha(e, 1)$ and the following diagram is commutative.

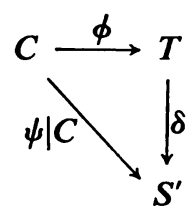

Now suppose $\delta(r)=\delta(s)$ for some $r, s \in T$. Then $((1, r),(1, s)) \in \mathscr{C}$. If $((1, r),(1, s)) \in \mathscr{P}$ there exists $a_{1}, a_{2}, a_{3} \in C$ such that $a_{1} \leqq r \leqq a_{2}, a_{1} \leqq s \leqq a_{3}, a_{1} 1=a_{2} 1=a_{3} 1$, and $a_{1} r=a_{1} s$. Hence, $a_{1}=a_{2}=a_{3}$ and $r$ and $s$ are in the same $\mathscr{H}$ class as the idempotent $a_{1}$. Therefore, $a_{1}$ acts as an identity for $r$ and $s$, and it follows that $r=s$. If $((1, r),(1, s)) \in \mathcal{Q}$, then again $r=s$. Thus, $\delta$ is an iseomorphism from $T$ onto $S^{\prime}$, and it follows immediately that $\left(S^{\prime}, \psi \mid C\right)$ is a semigroup through $C$ which is iseomorphic to $(T, \phi)$.

That $\psi(X)$ is contained in the centralizer of $S^{\prime}$ follows immediately from the fact that $\beta(X)$ is contained in the centralizer of $\{1\} \times T$. Finally, $S=\psi(X) S^{\prime}$ since $X \times T=\beta(X)(\{1\} \times T)$.

Therefore, $\left(S, S^{\prime}, \psi\right)$ is an object in the category $\mathscr{C}(X, C, T, \phi)$. It remains to be shown that $\left(S, S^{\prime}, \psi\right)$ is a universal object in the category.

Fix an object $\left(S_{1}, S_{1}^{\prime}, \psi_{1}\right)$ in $\mathscr{C}(X, C, T, \phi)$. Let $\eta$ be an iseomorphism from $(T, \phi)$ onto $\left(S_{1}^{\prime}, \psi_{1} \mid C\right)$. Then, the following diagram is commutative.

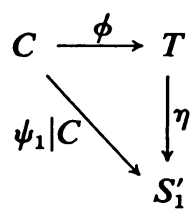

Define $\gamma: X \times T \rightarrow S_{1}$ by $\gamma(f, r)=\psi_{1}(f) \eta(r)$. Then $\gamma$ is a homomorphism since $\psi_{1}(X)$ is contained in the centralizer of $S_{1}^{\prime}$. We now show that there is an induced homomorphism $\theta: S \rightarrow S_{1}$ such that the following diagram is commutative.

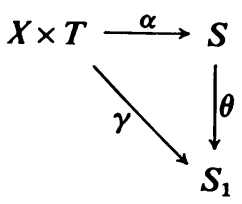

To avoid notational difficulties, $\psi_{1}(f)$ and $\eta(r)$ will be denoted by $\bar{f}$ and $\bar{r}$, respectively. We continue to identify $C$ with $\phi(C)$ in $T$. If $e \in X \cap T$ then $e \in C$ and $\psi_{1}(e)=\eta(e)$. Hence, there is no inconsistency in the use of this notation. 
Suppose $((f, r),(g, s)) \in \mathscr{P}$. Then, there exist $a_{1}, a_{2}, a_{3} \in C$ such that $a_{1} \leqq r \leqq a_{2}$, $a_{1} \leqq s \leqq a_{3}, a_{1} f=a_{2} f=a_{1} g=a_{3} g$, and $a_{1} r=a_{1} s$. Since $\psi_{1}(X)$ is contained in the centralizer of $S_{1}^{\prime}$,

$$
\bar{a}_{1} \bar{f} \leqq \bar{f} \bar{r} \leqq \bar{a}_{2} \bar{f} \text { and } \bar{a}_{1} \bar{g} \leqq \bar{g} \bar{s} \leqq \bar{a}_{3} \bar{g} \text {. }
$$

But $\bar{a}_{1} \bar{f}=\bar{a}_{2} \bar{f}=\bar{a}_{1} \bar{g}=\bar{a}_{3} \bar{g}$, so that $\bar{f} \bar{r}$ and $\bar{g} \bar{s}$ are in the same $\mathscr{H}$ class as $\bar{a}_{1} \bar{f}$. Now, $\bar{a}_{1}$ is an idempotent and it acts as an identity for $\bar{a}_{1} \bar{f}$. Hence, $\bar{a}_{1}$ acts as an identity for the $\mathscr{H}$ class of $\bar{a}_{1} \bar{f}$. It follows that

$$
\begin{array}{rlr}
\bar{f} \bar{r}=\bar{f} \bar{r} \bar{a}_{1} & =\bar{f} \bar{s} \bar{a}_{1} & \text { since } a_{1} r=a_{1} s \\
& =\bar{g} \bar{s} \bar{a}_{1} & \text { since } a_{1} f=a_{1} g \\
& =\bar{g} \bar{s} .
\end{array}
$$

Therefore, if $((f, r),(g, s)) \in \mathscr{P}$ then $\gamma(f, r)=\gamma(g, s)$.

Now suppose $((f, r),(g, s)) \in \mathscr{Q}$. Then $r=s$ and there exists $a \in C$ such that $a \geqq r$ and $a f=a g$. From $a \geqq r$ it follows that $a r=r$ so that $\bar{a} \bar{r}=\bar{r}$. Now $\bar{f} \bar{a}=\bar{g} \bar{a}$ implies $\bar{f} \bar{a} \bar{r}=\bar{g} \bar{a} \bar{r}$ which in turn implies $\bar{f} \bar{r}=\bar{g} \bar{r}$. Therefore, if $((f, r),(g, s)) \in \mathscr{Q}$ then $\gamma(f, r)=\gamma(g, s)$.

From the two previous paragraphs, the existence of the desired homomorphism $\theta$ is assured. We now show that $\theta$ is a morphism in the category $\mathscr{C}(X, C, T, \phi)$.

For the remainder of the proof we return to the functional notation. For each $f \in X$

$$
(\gamma \circ \beta)(f)=\gamma(f, 1)=\psi_{1}(f) \eta(1)=\psi_{1}(f)
$$

so that the following diagram is commutative.

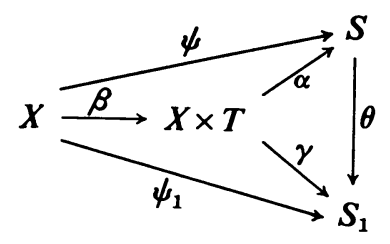

It remains to be shown that $\theta \mid S^{\prime}$ is an iseomorphism from $\left(S^{\prime}, \psi \mid C\right)$ onto $\left(S_{1}^{\prime}, \psi_{1} \mid C\right)$. In view of the two commuting diagrams
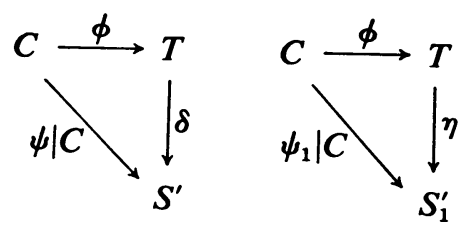

it suffices to show that $\theta \mid S^{\prime}=\eta \circ \delta^{-1}$. Fix $s \in S^{\prime}$ and let $r \in T$ such that $\alpha(1, r)=s$. Then, by definition of $\delta, \delta(r)=s$ so that $r=\delta^{-1}(s)$. Now,

$$
\theta(s)=(\theta \circ \alpha)(1, r)=\gamma(1, r)=\psi_{1}(1) \eta(r)=\eta(r)=\left(\eta \circ \delta^{-1}\right)(s)
$$

and $\theta \mid S^{\prime}=\eta \circ \delta^{-1}$. 
Hence, $\theta$ is a homomorphism from $\left(S, S^{\prime}, \psi\right)$ into $\left(S_{1}, S_{1}^{\prime}, \psi_{1}\right)$ such that $\theta \mid S^{\prime}$ is an iseomorphism from $\left(S^{\prime}, \psi \mid C\right)$ onto $\left(S_{1}^{\prime}, \psi_{1} \mid C\right)$. Therefore, $\left(S, S^{\prime}, \psi\right)$ is a universal object in $\mathscr{C}(X, C, T, \phi)$ and the proof of Theorem 4.1 is complete.

Proof of Corollary 4.1.1. This is an obvious corollary of the theorem and Theorem 3.1.

Proof of Corollary 4.1.2. This follows from the theorem, the obvious fact that (Horm $\left.\left(C, S_{x}, m_{x y}\right), \phi\right)$ is a semigroup straight through $C$, and the next theorem.

THEOREM 4.2. If $(T, \phi)$ is a semigroup straight through $C$, then each object in $\mathscr{C}(X, C, T, \phi)$ is a semigroup straight through $X$ relative to $C$.

Proof. Suppose $\left(S, S^{\prime}, \psi\right)$ is an object in $\mathscr{C}(X, C, T, \phi)$. As in the proof of Theorem 4.1, define $\beta: X \rightarrow X \times T$ and $\alpha: X \times T \rightarrow S$ by

$$
\beta(f)=(f, 1), \quad \alpha(f, r)=\psi(f) \delta(r)
$$

where $\delta$ is an iseomorphism from $(T, \phi)$ onto $\left(S^{\prime}, \psi \mid C\right)$. If $e \in E(S)$ then there exists $h \in E(X \times T)=X \times E(T)$ such that $\alpha(h)=e$. Hence, $h=(f, g)$ for some $f \in X$ and $g \in E(T)$. But $E(T)=\phi(C)$ since $(T, \phi)$ is straight through $C$ so that $g=\phi(k)$ for some $k \in C$. Therefore,

$$
\begin{aligned}
e=\alpha(h)=\alpha(f, g) & =\psi(f) \delta(g) \\
& =\psi(f)(\delta \circ \phi)(k) \\
& =\psi(f) \psi(k) \\
& =\psi(f k) \in \psi(X) .
\end{aligned}
$$

Hence, $\psi(X)=E(S)$. Since $\left(S^{\prime}, \psi \mid C\right)$ is a semigroup straight through $C$, it follows that $\psi(C)=E\left(S^{\prime}\right)$ and the proof is complete.

THEOREM 4.3. If $(T, \phi)$ is a clan through $C$ then each object in $\mathscr{C}(X, C, T, \phi)$ is a clan through $X$ relative to $C$.

Proof. Let $\left(S, S^{\prime}, \psi\right)$ be an object in $\mathscr{C}(X, C, T, \phi)$. Then $S^{\prime}$ is connected since it is homeomorphic to $T$. Given any $f \in X, \psi(f) S^{\prime}$ is connected and contains $\psi(z)$ since $\psi(z) \in S^{\prime}$. Therefore, $\bigcup\left\{\psi(f) S^{\prime}: f \in X\right\}=\psi(X) S^{\prime}=S$ is connected and the proof is complete.

5. Universal objects in other categories.

Definition 5.1. We will denote by $\mathscr{C}(C)$ the category whose objects are clans straight through $C$. The morphisms are homomorphisms as defined in Definition 3.2.

Definition 5.2. We will denote by $\mathscr{C}(X, C)$ the category whose objects are clans straight through $X$ relative to $C$. The morphisms are homomorphisms as defined in Definition 4.2.

THEOREM 5.1. There exists a universal object in $\mathscr{C}(C)$. 
Proof. By Theorems 5.25 to 5.29, pages $152-155$ of [2] there exists an irreducible clan $(\operatorname{Irr} C, \phi)$ straight through $C$ such that if $(S, \psi)$ is any irreducible clan straight threugh $C$, then there exists a continuous homomorphism $\alpha$ from $(\operatorname{Irr} C, \phi)$ onto $(S, \psi)$. Now if $(T, \eta)$ is a clan straight through $C$, let $S$ be an irreducible subclan of $T$ containing the identity of $T$ and the idempotent in the minimal ideal of $T$. For each $e \in \eta(C \mid\{z, 1\}), H(e)$ separates $T$ (see (b) of Definition 3.1). Hence, as $S$ is connected and contains both $\eta(z)$ and $\eta(1), e \in S$ for each $e \in \eta(C \mid\{z, 1\})$. We define $\psi: C \rightarrow S$ by $\psi(f)=\eta(f)$. Clearly $(S, \psi)$ satisfies (a) of Definition 3.1. That (b) of Definition 3.1 is satisfied for $(S, \psi)$ follows from the fact that any irreducible semigroup is totally quasi-ordered relative to $\leqq[2$, Theorem IV, p. 132]. So $(S, \psi)$ is a clan through $C$. Moreover, $(S, \psi)$ is a clan straight through $C$ since $(T, \eta)$ is. Hence there exists $\alpha$ as above from $(\operatorname{Irr} C, \phi)$ onto $(S, \psi)$. It follows that $i \circ \alpha$ is a homomorphism from $(\operatorname{Irr} C, \phi)$ into $(T, \eta)$ where $i$ is the injection from $S$ into $T$. Hence, $(\operatorname{Irr} C, \phi)$ is the desired universal object.

THEOREM 5.2. There exists a universal object in $\mathscr{C}(X, C)$.

Proof. Let $\left(S, S^{\prime}, \psi\right)$ be the universal object in the category $\mathscr{C}(X, C, \operatorname{Irr} C, \phi)$ constructed in the proof of Theorem 4.1 (where $(\operatorname{Irr} C, \phi)$ is the universal object of $\mathscr{C}(C)$ obtained in the proof of Theorem 5.1). We show that $\left(S, S^{\prime}, \psi\right)$ is a universal object in $\mathscr{C}(X, C)$. That $\left(S, S^{\prime}, \psi\right)$ is a clan straight through $X$ relative to $C$ follows from Theorems 4.2 and 4.3. Let $\left(S_{1}, S_{1}^{\prime}, \psi_{1}\right)$ be an arbitrary object in $\mathscr{C}(X, C)$. Let $T_{1}^{\prime}$ be an irreducible semigroup in $S_{1}^{\prime}$ containing $\psi_{1}(1)$ and $\psi_{1}(z)$. Then, as in the proof of Theorem 5.1, $\left(T_{1}^{\prime}, \psi_{1} \mid C\right)$ is a clan straight through $C$. Hence, by Theorem 5.1, there exists a continuous homomorphism $\eta$ from $(\operatorname{Irr} C, \phi)$ onto $\left(T_{1}^{\prime}, \psi_{1} \mid C\right)$. As in the proof of Theorem 4.1, let $\alpha$ be the natural map from $X \times \operatorname{Irr} C$ onto $S$; let $\beta: X \rightarrow X \times \operatorname{Irr} C$ be defined by $\beta(f)=(f, 1)$; and let $\gamma: X \times \operatorname{Irr} C \rightarrow S_{1}$ be defined by $\gamma(f, r)=\psi_{1}(f) \eta(r)$. Again, the methods of the proof of Theorem 4.1 give the existence of a continuous homomorphism $\theta: S \rightarrow S_{1}$ such that the following diagram is commutative:

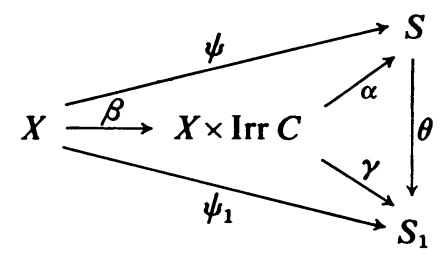

It is easily seen that $\theta \mid S^{\prime}$ is a homomorphism from $\left(S^{\prime}, \psi \mid C\right)$ into $\left(S_{1}^{\prime}, \psi_{1} \mid C\right)$. This completes the proof.

6. Minimality conditions. One shortcoming of Theorems 5.1 and 5.2 is that the morphisms from the universal objects to other objects need not be surmorphisms. Clearly, any morphism from (Irr $C, \phi)$ to an irreducible object of $\mathscr{C}(C)$ is a surmorphism. We now consider the analagous result for $\mathscr{C}(X, C)$. 
Definition 6.1. Let $S$ be a clan and let $A$ be a subset of $S$. Then $S$ is minimal about $A$ if no proper subclan of $S$ contains $A$. If $S$ is minimal about $E(S)$ then $S$ is called minimal about its idempotents. (By a subclan we mean a clan contained in $S$ with the same identity as $S$.)

Note. A simple maximality argument yields that each clan $S$ contains a subclan minimal about a fixed subset of $S$.

The proofs of the following two theorems are straightforward and will be omitted.

Theorem 6.1. Let $\left(S, S^{\prime}, \psi\right)$ and $\left(S_{1}, S_{1}^{\prime}, \psi_{1}\right)$ be objects of $\mathscr{C}(X, C)$. If $S_{1}$ is minimal about its idempotents then any morphism from $\left(S, S^{\prime}, \psi\right)$ to $\left(S_{1}, S_{1}^{\prime}, \psi_{1}\right)$ is a surmorphism.

THEOREM 6.2. Let $S$ be an abelian clan, minimal about its idempotents, and let $\psi$ be the injection of $E(S)$ into $S$. Let $T$ be a subclan of $S$ containing the idempotent of the minimal ideal of $S$ such that $(T, \psi \mid E(T))$ is a semigroup through $E(T)$. Then $(S, T, \psi)$ is an object of both $\mathscr{C}(E(S), E(T), T, \phi)$ and $\mathscr{C}(E(S), E(T))$, where $\phi$ is the injection of $E(T)$ into $T$.

THEOREM 6.3 (HoFMANN AND MOSTERT). Let $S$ be a clan with abelian set of idempotents. Let 1 be the identity of $S$ and let $z$ be the idempotent in the minimal ideal of $S$. If $S$ is minimal about $\{1, z\}$ then $S$ is abelian.

One might reasonably ask the question "If $S$ is a clan with abelian set of idempotents and $S$ is minimal about its idempotents, must $S$ be abelian?" The following example answers this question in the negative.

EXAMPLE 6.1. In [1, p. 41], Brown defines a semilattice multiplication on the 2-cell $D$ in such a way that the circle group $G$ acts as a group of automorphisms of $D$ under rotation. If $D^{\prime}$ is $D$ with a discrete identity $e$ adjoined, then $G$ acts on $D^{\prime}$ by rotation on $D$ and trivial action on $e$. It follows that $G$ acts on $D^{\prime} \times[0,1]$ and hence on $T=\left(D^{\prime} \times[0,1]\right) /\left(D^{\prime} \times\{0\}\right)$ where $[0,1]$ has min multiplication. Note that $T$ is a semilattice. By the remark on page 27 of [2], Split $(G, T)$ is not abelian. However, Split $(G, T)$ is not minimal about its idempotents. The element $\phi(e, 1)$ is the identity of Split $(G, T)$ where $\phi$ is the natural map from $D^{\prime} \times[0,1]$ onto $T$ and according to Theorem 2.32 of [2, page 27], the group of units of Split $(G, T)$ is $G \times\{\phi(e, 1)\}$. Let $S$ be the semigroup obtained by sewing the minimal ideal of a one-parameter semigroup winding on the circle [3, p. 286] to $G \times\{\phi(e, 1)\}$ in $T$ and multiplying in the obvious manner. (This semigroup $S$ is iseomorphic to GHorm $\left(X, S_{x}, m_{x y}\right)$ where $X=\{0, e, 1\}, S_{0}=G, S_{e}=\operatorname{Split}(G, T), S_{1}$ is the oneparameter semigroup winding on the circle, and $m_{x y}$ is the obvious homomorphism for each $x \leqq y$.) Then, it is not difficult to show that $S$ is a clan with abelian set of idempotents and $S$ is minimal about its idempotents. However, $S$ is not abelian.

Finally, we pose the following question raised by C. E. Clark in an oral communication: If $S$ is a clan with abelian set of idempotents, $S$ is minimal about its idempotents, and $E(S)$ is a chain, must $S$ be abelian? 


\section{REFERENCES}

1. D. R. Brown, Topological semilattices on the two-cell, Pacific J. Math. 15 (1965), 35-46. MR 31 \#725.

2. K. H. Hofmann and P. S. Mostert, Elements of compact semigroups, Charles E. Merrill, Columbus, Ohio, 1966. MR 35 \#285.

3. R. J. Koch and A. D. Wallace, Admissibility of semigroup structures on continua, Trans. Amer. Math. Soc. 88 (1958), 277-287. MR 20 \#1729.

4. Barry Mitchell, Theory of categories, Pure and Appl. Math., vol. 17, Academic Press, New York, 1965. MR 34 \#2647.

UNIVERSITY OF TENNESSEE,

KNOXVILle, Tennessee 37916 